\title{
Detecting Rashba fields at the interface between Co and Si oxide by ferromagnetic resonance
}

\author{
T. G. A. Verhagen, I. Leermakers, J. M. van Ruitenbeek, and J. Aarts* \\ Huygens-Kamerlingh Onnes Laboratorium, Universiteit Leiden, P.O. Box 9504, 2300 RA Leiden, The Netherlands
}

(Received 29 June 2014; revised manuscript received 19 April 2015; published 7 May 2015)

\begin{abstract}
We report ferromagnetic resonance (FMR) experiments on thin magnetic Co layers either sandwiched symmetrically between $\mathrm{Cu}$ and $\mathrm{Pt}$, or sandwiched asymmetrically between a $\mathrm{Cu}$ or $\mathrm{Pt}$ layer on one side and a $\mathrm{SiO}_{x}$ substrate on the other. In the symmetric samples, we find well-known behavior, namely, the FMR linewidth $\Delta H_{p p}$ is significantly larger for the case of $\mathrm{Pt}$ than for the case of $\mathrm{Cu}$. This is due to the larger spin scattering in the Pt layer. However, for the asymmetric $\mathrm{Co} / \mathrm{Cu}$ bilayers, the linewidth is much larger than for the symmetric $\mathrm{Cu} / \mathrm{Co} / \mathrm{Cu}$ trilayers and not much different from the linewidth of $\mathrm{Co} / \mathrm{Pt}$ bilayers. We argue this to be due to the Rashba effect at the $\mathrm{SiO}_{x} / \mathrm{Co}$ interface, which gives rise to effective magnetic fields interacting with the electron spins in the Co layer and which can be measured without reverting to transport measurements.
\end{abstract}

DOI: 10.1103/PhysRevB.91.184402

PACS number(s): 75.76.+j, 75.70.-i, 76.50.+g

\section{INTRODUCTION}

Traditional spintronic devices consist of two ferromagnetic layers with different magnetization directions, the polarizer and the free layer, with some spacer in between. When flowing a large enough current through the device, the magnetization direction of the free layer can be changed via the spin transfer torque mechanism, thereby giving rise to electrical switching of the magnetization.

Recently, experiments and theory have shown that there are alternative mechanisms that can produce a spin torque, based on the spin-orbit interaction (SOI), which transfers orbital angular momentum from the lattice to the spin system. Two effects based on the SOI are the spin Hall and the Rashba effects, and both are currently investigated with respect to their potential to generate a spin-orbit torque (SOT). This would provide an alternative and efficient way for electric magnetization switching, since only one ferromagnetic layer is required.

Spin-orbit torques can be related to lack of inversion symmetry either in the bulk of a material or in thin film structures, resulting in a Rashba or a Dresselhaus spin-orbit coupling, respectively. Well known systems where such a lack of bulk inversion symmetry can be found are semiconductors with the zinc blende structure such as (Ga,Mn)As [1-3] or crystals from the B20 space group such as FeGe [4] and MnSi [5], which show a chiral spin-orbit interaction, described by the Dzyaloshinsky-Moriya (DM) interaction. As a consequence, nontrivial spin structures can occur in these systems.

Inversion asymmetry can also be engineered. In recent ac magnetotransport experiments, the ferromagnetic layer was asymmetrically sandwiched between a heavy metal layer and an oxide layer, e.g., $\mathrm{Pt} / \mathrm{Co} / \mathrm{AlOx}$ or $\mathrm{Ta} / \mathrm{CoFeB} / \mathrm{MgO}$ [6-13]. Measuring the harmonic and second harmonic Hall voltages allows then to find values for Rashba fields [8] and the various torques that act on the electron spin in consequence [13]. Concentrating on the Rashba field $H_{\text {ra }}$, according to Ref. [8], this is given by

$$
H_{\mathrm{ra}}=\frac{\alpha_{R} J_{s d}}{\mu_{B} M_{s}}(\hat{z} \times \boldsymbol{J}),
$$

\footnotetext{
*aarts@physics.leidenuniv.nl
}

where $\alpha_{R}$ is the strength of the Rashba interaction, $M_{S}$ is the saturation magnetization, $\mu_{B}$ is the Bohr magneton, and $J_{s d}$ measures the s-d coupling strength. The direction $\hat{z}$ is perpendicular to the interfaces and $\boldsymbol{J}$ is the in-plane current density, making the effective field $H_{\mathrm{ra}}$ a transverse field. Described in this way, fields and torques are only nonzero in the presence of a net charge transport current, which then also defines their direction. Without a net current, the average Rashba field will be zero, but its presence may still be experienced in the random motion of the electrons.

Here, we search for such a signature by performing a ferromagnetic resonance (FMR) experiment, using FMR to inject a spin current into a $\mathrm{Cu}$ layer by spin pumping from a Co layer [14]. We observe significantly larger damping in a $\mathrm{SiO}_{x} / \mathrm{Co}(d) / \mathrm{Cu}$ bilayer than in $\mathrm{Cu} / \mathrm{Co}(d) / \mathrm{Cu}$ trilayers, and comparable to the damping observed in $\mathrm{Co}$ bi- and trilayers capped with $\mathrm{Pt}$, which has a strong spin-orbit coupling. We attribute this to the presence of an effective magnetic field arising from the extra spin-orbit torque induced by the broken inversion symmetry at the $\mathrm{SiO}_{x} / \mathrm{Co}$ interface. The FMR experiment allows to observe this effective field even in the absence of a transport current.

\section{EXPERIMENTAL DETAILS}

The following films were grown: $\operatorname{Co}(d) / \operatorname{Pt}(10), \operatorname{Pt}(10) /$ $\mathrm{Co}(d) / \mathrm{Pt}(10), \mathrm{Co}(d) / \mathrm{Cu}(10)$, and $\mathrm{Cu}(10) / \mathrm{Co}(d) / \mathrm{Cu}(10)$. The numbers in parentheses represent the layer thickness in nanometers; $d$ ranges between 1.6 and $10.5 \mathrm{~nm}$. The films were deposited at room temperature on naturally oxidized $\mathrm{Si}(100)$ in a UHV chamber (base pressure $1 \times 10^{-9}$ mbar) using DC magnetron sputter deposition with an argon plasma from 3N5 Co, 3N5 Cu, and 3N5 Pt targets. To emphasize the interfaces of the Co layer, we describe the $\mathrm{Co} / \mathrm{Pt}$ and $\mathrm{Co} / \mathrm{Cu}$ bilayers as $\mathrm{SiO}_{x} / \mathrm{Co} / \mathrm{Cu}$ and $\mathrm{SiO}_{x} / \mathrm{Co} / \mathrm{Pt}$. Also, since the thicknesses of the $\mathrm{Cu}$ - and Pt-layers are not going to be varied, we use the notation $\operatorname{Co}(d) / \mathrm{Pt}$ etc. The deposition rate was measured by $\mathrm{x}$-ray reflectivity (XRR) using $\mathrm{Cu}-\mathrm{K} \alpha$ radiation and was $0.58 \AA$ /s for $\mathrm{Co}, 1.90 \AA / \mathrm{s}$ for $\mathrm{Cu}$, and $1.54 \AA / \mathrm{s}$ for $\mathrm{Pt}$, respectively. Magnetization measurements were performed using the reciprocating sample option (RSO) in a SQUIDbased magnetometer (MPMS XL-7 from Quantum Design). Ferromagnetic resonance (FMR) was measured using a Bruker 

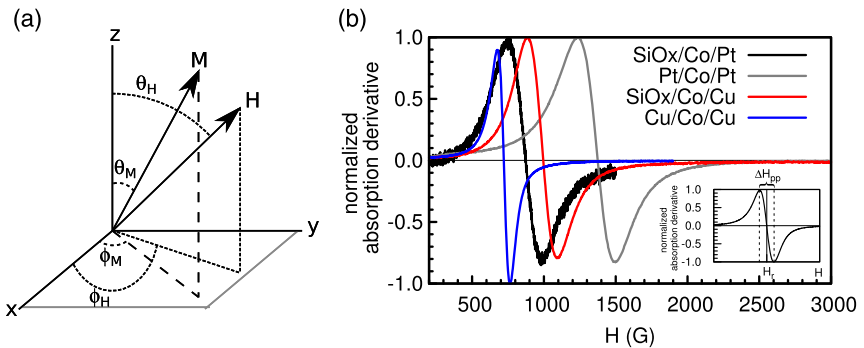

FIG. 1. (Color online) (a) The coordinate system used for the analysis of the FMR data. The film lies in the $x y$ plane and its magnetization $M$ points in the $M$ direction. The applied dc magnetic field $H$ points in the $H$ direction. (b) The in-plane $\left(\theta_{H}=90^{\circ}\right)$ FMR spectra of $\mathrm{Cu} / \mathrm{Co}(2.6) / \mathrm{Cu}$ (blue, left), $\mathrm{SiO}_{x} / \mathrm{Co}(2.6) / \mathrm{Pt}$ (black), $\mathrm{SiO}_{x} / \mathrm{Co}(2.6) / \mathrm{Cu}$ (red), and $\mathrm{Pt} / \mathrm{Co}(2.6) / \mathrm{Pt}$ (gray, right) multilayers. The inset shows the normalized derivative absorption spectrum as a function of the applied static magnetic field $H$, with the resonance field $H_{r}$, and the peak-to-peak linewidth $\Delta H_{p p}$ indicated.

EMXplus X-band $(9.8 \mathrm{GHz})$ spectrometer in a $\mathrm{T} E_{011}$ cavity with $100 \mathrm{kHz}$ modulation frequency and $0.1 \mathrm{mT}$ modulation amplitude with a maximum dc field of $0.65 \mathrm{~T}$. The sample was fixed on a Rexolite 1422 rod and a goniometer was used to vary the angle. The measurements were performed at room temperature.

From the FMR spectra, static and dynamic properties of thin magnetic layers can be obtained [15], in particular the intrinsic relaxation of the magnetization parametrized by the Gilbert damping parameter $\alpha$. For this, either the frequency or the angular dependence can be used [16,17]. Here we use the latter. The angular dependent resonance condition is [16]

$$
\begin{gathered}
\left(\frac{\omega}{\gamma}\right)^{2}=H_{1} \times H_{2}, \\
H_{1}=H_{r} \cos \left(\theta_{H}-\theta_{M}\right)-4 \pi M_{\text {eff }} \cos ^{2} \theta_{M}, \\
H_{2}=H_{r} \cos \left(\theta_{H}-\theta_{M}\right)-4 \pi M_{\text {eff }} \cos 2 \theta_{M} .
\end{gathered}
$$

Here, $\omega$ is the angular frequency of the microwaves, and $\gamma=$ $g \mu_{B} / \hbar$ the gyromagnetic ratio entailing the electron $g$ factor; $H_{r}$ is the resonance field, $\theta_{H / M}$ are the polar angles of the dc magnetic field $H$ and the magnetization $M$ [see the coordinate system in Fig. 1(a)]; $4 \pi M_{s}$ is the saturation magnetization and $4 \pi M_{\text {eff }}=4 \pi M_{s}-H_{\perp}$ is the effective demagnetization field with $H_{\perp}=2 K_{\perp} / M_{s}$, where $K_{\perp}$ is the perpendicular magnetic anisotropy (PMA).

The peak-to-peak linewidth $\Delta H_{p p}$ of the FMR spectra is determined by the different damping mechanisms present in the magnetic multilayer. Intrinsic relaxation of the magnetization leads to a homogeneous broadening of the linewidth $\Delta H_{p p}^{\text {homo }}$, which is given by $[16,18]$

$$
\Delta H_{p p}^{\mathrm{homo}}=\frac{1}{\sqrt{3}} \frac{\alpha}{M_{s}}\left(H_{1}+H_{2}\right)\left|\frac{d(\omega / \gamma)}{d H_{r}}\right|^{-1},
$$

where $\alpha$ is the Gilbert damping. If local inhomogeneities in the thin film are not negligible this can cause extra line broadening
$\Delta H_{p p}^{\text {inhomo }}$, which can be described by $[19,20]$

$$
\begin{aligned}
\Delta H_{p p}^{\text {inhomo }}= & \frac{1}{\sqrt{3}}\left|\frac{d H_{r}}{d\left(4 \pi M_{\mathrm{eff}}\right)}\right| \Delta\left(4 \pi M_{\mathrm{eff}}\right) \\
& +\frac{1}{\sqrt{3}}\left|\frac{d H_{r}}{d \theta_{H}}\right| \Delta\left(\theta_{H}\right),
\end{aligned}
$$

where $\Delta\left(\theta_{H}\right)$ and $\Delta\left(4 \pi M_{\text {eff }}\right)$ are the spatial variations in the spread of the crystallographic axis and the demagnetization field, respectively.

\section{RESULTS}

In Fig. 1(b), in-plane $\left(\theta_{H}=90^{\circ}\right)$ FMR spectra of $\mathrm{Cu} / \mathrm{Co}(2.6) / \mathrm{Cu}$ and $\mathrm{Pt} / \mathrm{Co}(2.6) / \mathrm{Pt}$ trilayers and $\mathrm{SiO}_{x} / \mathrm{Co}(2.6) / \mathrm{Cu}$ and $\mathrm{SiO}_{x} / \mathrm{Co}(2.6) / \mathrm{Pt}$ bilayers are plotted. In Fig. 2(a), the angular dependent peak-to-peak linewidth $\Delta H_{p p}$ of these four samples is shown. Clearly visible is that for all angles $\Delta H_{p p}$ of the $\mathrm{Cu} / \mathrm{Co} / \mathrm{Cu}$ trilayer is much smaller than $\Delta H_{p p}$ of the $\mathrm{Pt} / \mathrm{Co} / \mathrm{Pt}$ and $\mathrm{SiO}_{x} / \mathrm{Co} / \mathrm{Pt}$ films, but also much smaller than $\Delta H_{p p}$ of the $\mathrm{SiO}_{x} / \mathrm{Co} / \mathrm{Cu}$ bilayer. In Fig. 2(b), the angular dependent resonant fields $H_{r}$ are plotted. $H_{r}$ of the $\mathrm{Pt} / \mathrm{Co} / \mathrm{Pt}$ trilayer is somewhat larger than $H_{r}$ of the other trilayers, for which $H_{r}$ is almost the same.

Before analyzing these data further, we want to address the question whether the extra damping can simply be caused by magnetic impurities in the nonmagnetic layer. For this we used the $\delta$-doping technique as was done before by Marrows and Hickey [21] to investigate the role of impurities in GMR systems. With $\delta$ doping, a very thin magnetic layer, in our case of $\mathrm{Co}$, is added somewhere in the nonmagnetic layer. Specifically, we grew $\mathrm{Cu} / \mathrm{Co}(3.6) / \mathrm{Cu}(5) / \mathrm{Co}\left(\delta_{\mathrm{Co}}\right) / \mathrm{Cu}(5)$

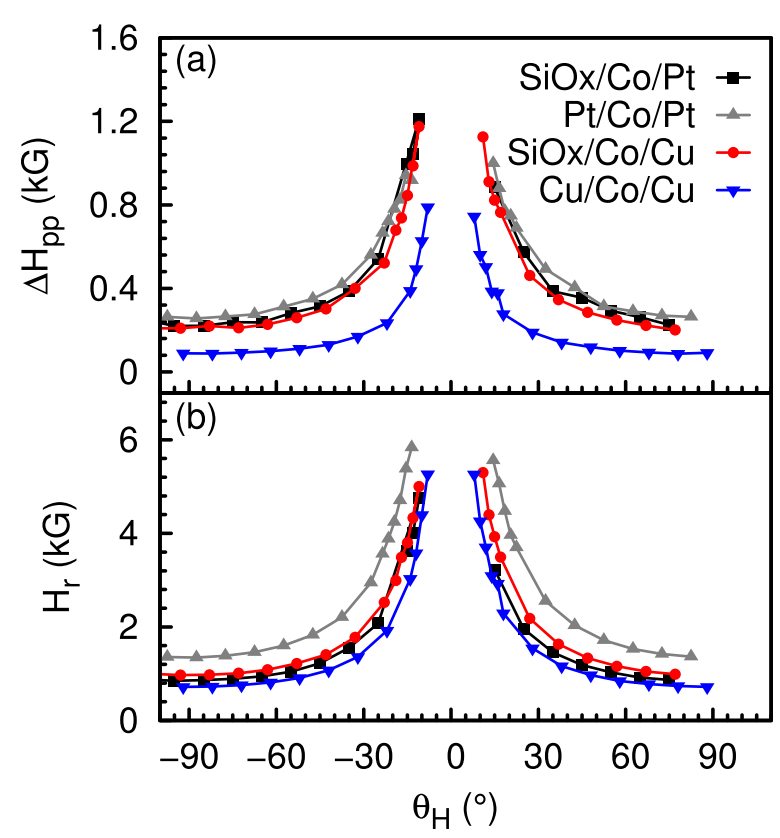

FIG. 2. (Color online) Angular dependence of the peak-to-peak linewidth $H_{p p}$ (a) and the resonance field $H_{r}$ (b) as a function of the applied field direction $\theta_{H}$ for a $\mathrm{SiO}_{x} / \mathrm{Co}(2.6) / \mathrm{Pt}$ (black $\mathbf{\square}$ ), $\mathrm{SiO}_{x} / \mathrm{Co}(2.6) / \mathrm{Cu}$ (red $\left.\bigcirc\right), \mathrm{Cu} / \mathrm{Co}(2.6) / \mathrm{Cu}$ (blue $\nabla$ ), and $\mathrm{Pt} / \mathrm{Co}(2.6) / \mathrm{Pt}$ (gray $\triangle$ ) multilayers. 


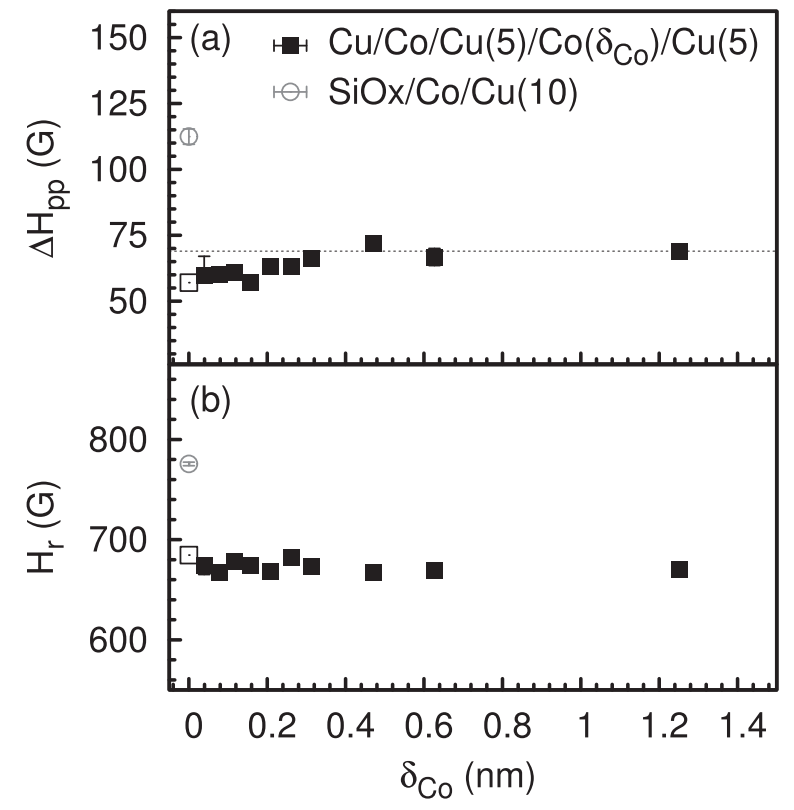

FIG. 3. Measurements with field in-plane $\left(\theta_{H}=90^{\circ}\right)$ of (a) the peak-to-peak linewidth $\Delta H_{p p}$ and (b) the resonance field $H_{r}$ for a $\mathrm{Cu} / \mathrm{Co}(3.6) / \mathrm{Cu}(5) \mathrm{Co}\left(\delta_{\mathrm{Co}}\right) / \mathrm{Cu}(5)$ multilayer as a function of the thickness of the Co impurity layer $\delta_{\mathrm{Co}}$ (black $\mathbf{\square}$, with the point $\delta_{\mathrm{Co}}=$ 0 indicated with a open square $\square$ ). For comparison, also $\Delta H_{p p}$ and $H_{r}$ of a $\mathrm{SiO}_{x} / \mathrm{Co}(3.6) / \mathrm{Cu}$ bilayer are plotted (gray $\bigcirc$ ). As a guide to the eye, the grey dotted line indicates the maximum $\Delta H_{p p}$ for the $\delta$-doping sample.

multilayers where the thickness of the $\delta_{\mathrm{Co}}$ impurity layer is varied between 0 and $1.2 \mathrm{~nm}$. For the growth of this very thin Co layers, a Co deposition rate of $0.13 \AA$ /s was used. Figure $3\left(\right.$ a) shows $\Delta H_{p p}$ of $\mathrm{Cu} / \mathrm{Co}(3.6) / \mathrm{Cu}(5) / \mathrm{Co}\left(\delta_{\mathrm{Co}}\right) / \mathrm{Cu}(5)$ multilayers as a function of the Co impurity layer $\delta_{\mathrm{Co}}$, measured with the field in the plane of the layers $\left(\theta_{H}=90^{\circ}\right)$. We find that, as the thickness of the impurity layer increases, $\Delta H_{p p}$ starts to increase from its value of $60 \mathrm{G}$ and saturates already for a $0.4-\mathrm{nm}$ thick impurity layer at a value around 70 G. In Fig. 3(b), we plot $H_{r}$ of these samples, which is almost constant for the whole impurity layer thickness range. It shows that the thickness of the 3.6-nm thick Co layer does not vary from sample to sample and that the Co impurity layer does not couple to the thick Co layer. Figure 3 also shows $\Delta H_{p p}$ and $H_{r}$ of the $\mathrm{SiO}_{x} / \mathrm{Co}(3.6) / \mathrm{Cu}$ bilayer. Both values are much larger than those of the $\delta$-doping sample, which strongly suggests that magnetic impurities are not the cause of the large increase of the damping in $\mathrm{SiO}_{x} / \mathrm{Co} / \mathrm{Cu}$ bilayers. The values found in $\delta=0$ sample once more stress the large differences we find between the bilayer and trilayer samples.

\section{ANALYSIS}

Returning to the bilayers, for all samples the saturation magnetization $M_{s}$ was obtained using a magnetometer. Together with the angular dependence of $\Delta H_{p p}$ it allows us to extract the values for the effective demagnetization $M_{\text {eff }}$ and for the damping $\alpha$. As we are not able to measure the full angular dependence due to the limitation on the dc field, we fit each curve with different $g$ factors between 1.8 and 2.2.
From the fits we extract the average, minimum, and maximum values, which are shown in the figures as "error bars." The range for the $g$ value is chosen between the bulk value for $\mathrm{Co}, 2.2$, and the best fitting $g$ value for the $\mathrm{SiO}_{x} / \mathrm{Co}(2) / \mathrm{Pt}$ bilayer, 1.8. A $g$ value of 1.8 may appear to be low, but both the induced orbital magnetization [22] and the spin pumping effect decrease the $g$ value. Zhang et al. [23], from FMR experiments on $\mathrm{Si} / \mathrm{Ru} / 20 \times[\mathrm{Co}(25 \AA)+\mathrm{Pt}(\mathrm{d})] / \mathrm{Pt}$ multilayers, found that for a varying Pt thickness $d_{\text {Co }}$ the $g$ value varied between 1.83 and 2.30 .

In Figs. 4(a)-4(d), we take a closer look at the magnetic properties of the multilayers. The saturation magnetization $4 \pi M_{s}$ [Figs. 4(a) and 4(b)], and the effective demagnetization fields $4 \pi M_{\text {eff }}$ [Figs. 4(c) and 4(d)], obtained from the analysis of the angular dependent FMR, are plotted as a function of the Co thickness $d_{\mathrm{Co}}$. The data for the Pt/Co(1.7)/Pt trilayer are not included in the analysis, as two resonance modes [23] were observed in this sample and the origin of the other mode is not clear.

For large Co thicknesses, $4 \pi M_{s}$ has reached the saturation magnetization value of bulk Co in all samples, as indicated by the horizontal dotted line. In the $\mathrm{Pt} / \mathrm{Co} / \mathrm{Pt}$ and $\mathrm{SiO}_{x} / \mathrm{Co} / \mathrm{Pt}$ samples, $4 \pi M_{s}$ is at the bulk saturation already also for small Co thicknesses. However, in the $\mathrm{Cu} / \mathrm{Co} / \mathrm{Cu}$ and $\mathrm{SiO}_{x} / \mathrm{Co} / \mathrm{Cu}$ samples, $4 \pi M_{s}$ becomes gradually smaller for thinner Co layers. For all samples, $4 \pi M_{\text {eff }}$ is lower than $4 \pi M_{s}$ of bulk Co. For the $\mathrm{Pt} / \mathrm{Co} / \mathrm{Pt}$ and $\mathrm{SiO}_{x} / \mathrm{Co} / \mathrm{Pt}$ samples, $4 \pi M_{\text {eff }}$ decreases rapidly with a decreasing Co thickness. $4 \pi M_{\text {eff }}$ decreases also for $\mathrm{Cu} / \mathrm{Co} / \mathrm{Cu}$ samples, but the change is much less than for the $\mathrm{SiO}_{x} / \mathrm{Co} / \mathrm{Pt}$ and $\mathrm{Pt} / \mathrm{Co} / \mathrm{Pt}$ multilayers. The $\mathrm{SiO}_{x} / \mathrm{Co} / \mathrm{Cu}$ bilayers show a decrease in $4 \pi M_{\text {eff }}$, which is equally strong as for $\mathrm{SiO}_{x} / \mathrm{Co} / \mathrm{Pt}$. This indicates that in both bilayers a PMA field of the same order of magnitude is present. With $M_{\text {eff }}$ and $M_{s}$ both available, we can evaluate $H_{\text {perp }}$ and $K_{\text {perp }}$, which will be done in Appendix A. In Figs. 4(e) and 4(f), the Gilbert damping $\alpha$ is plotted for the $\mathrm{Cu}-$ and Pt-based trilayers and bilayers, respectively. The value of $\alpha$ in the $\mathrm{Pt} / \mathrm{Co}\left(d_{\mathrm{Co}}\right) / \mathrm{Pt}$ trilayer increases rapidly with decreasing $d_{\mathrm{Co}}$, as $\mathrm{Pt}$ is a good spin sink. For the $\mathrm{Cu} / \mathrm{Co}\left(d_{\mathrm{Co}}\right) / \mathrm{Cu}$ trilayer, $\alpha$ is almost constant up to the lowest $\mathrm{Co}$ thickness, as $\mathrm{Cu}$ is a bad spin sink. The $\mathrm{SiO}_{x} / \mathrm{Co}\left(d_{\mathrm{Co}}\right) / \mathrm{Pt}$ bilayer shows also an increase in $\alpha$. However, although $\mathrm{Cu}$ is a bad spin sink, $\alpha$ of the $\mathrm{SiO}_{x} / \mathrm{Co}\left(d_{\mathrm{Co}}\right) / \mathrm{Cu}$ bilayer behaves nearly identical to the $\mathrm{SiO}_{x} / \mathrm{Co} / \mathrm{Pt}$ bilayer in contrast to what was seen in the trilayers. This unexpectedly large increase in the Gilbert damping in $\mathrm{SiO}_{x} / \mathrm{Co}\left(d_{\mathrm{Co}}\right) / \mathrm{Cu}$ bilayers with small $\mathrm{Co}$ thickness, together with the direct observation of much larger FMR linewidths are our main results, which need now to be discussed.

\section{DISCUSSION}

From the FMR spectra, an estimate can be made of extra damping fields acting on the system. Comparing the bilayers and trilayers, we see that $\Delta H_{p p}$ is larger by $113 \mathrm{G}$ in the $\mathrm{SiO}_{x} / \mathrm{Co}(2.6) / \mathrm{Cu}$ bilayer than in the $\mathrm{Cu} / \mathrm{Co}(2.6) / \mathrm{Cu}$ trilayer, which we take as the magnitude of extra effective dampinglike field. This is unexpected in the sense that the $\mathrm{Cu}$ layer, as a bad spin sink, is not supposed to generate a spin pumping effect as seen by the FMR line broadening. 

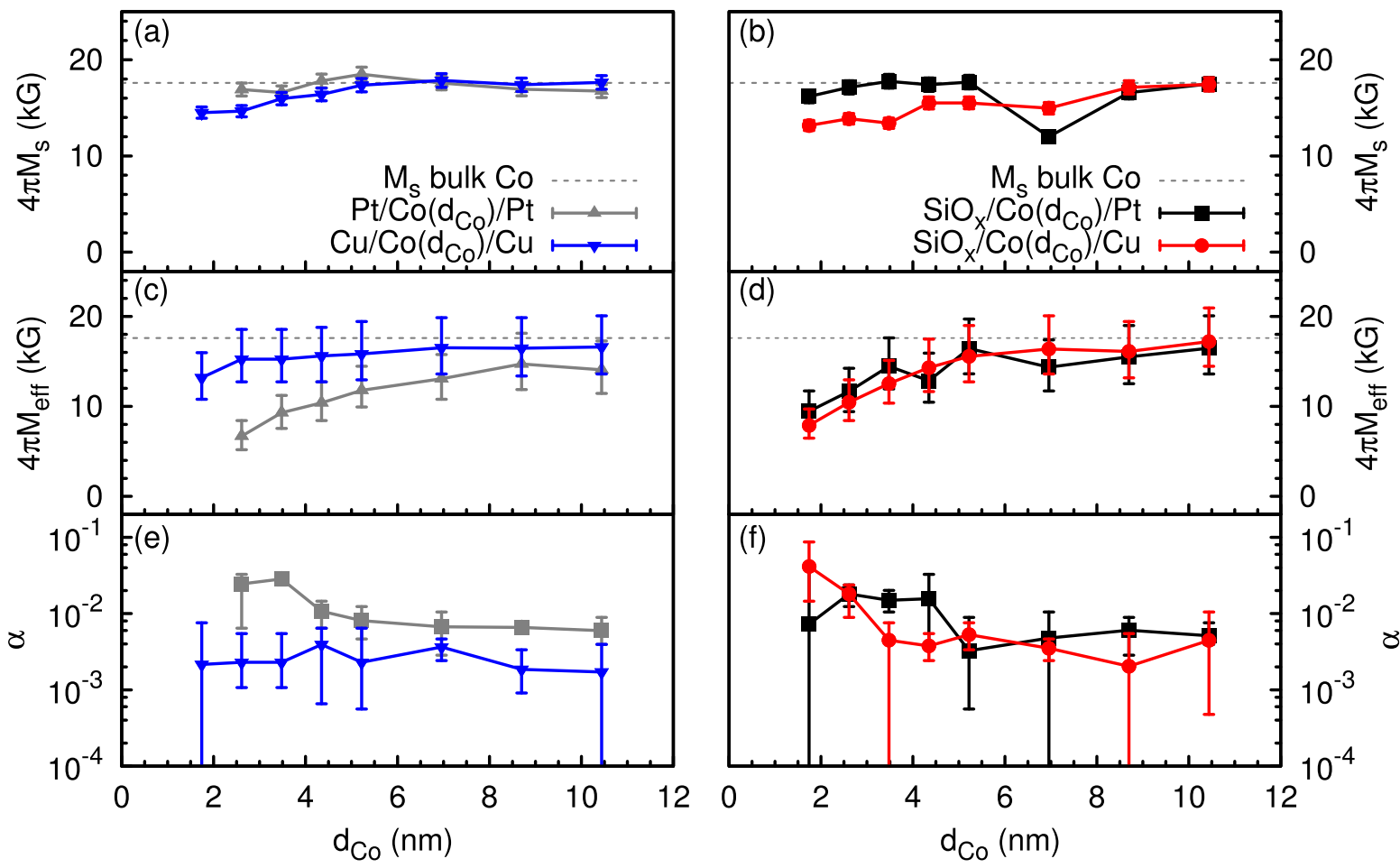

FIG. 4. (Color online) The saturation magnetization $4 \pi M_{s}$, effective demagnetization $4 \pi M_{\mathrm{eff}}$ and the damping $\alpha$, as a function of the thickness $d_{\mathrm{Co}}$ of the $\mathrm{Co}$ layer in the $\mathrm{Cu} / \mathrm{Co}\left(d_{\mathrm{Co}}\right) / \mathrm{Cu}$ (blue $)$ and $\mathrm{Pt} / \mathrm{Co}\left(d_{\mathrm{Co}}\right) / \mathrm{Pt}\left(\right.$ gray $\triangle$ ) trilayers $[(\mathrm{a}),(\mathrm{c})$, and $(\mathrm{e})]$ and in the $\mathrm{SiO} \mathrm{O}_{x} / \mathrm{Co}\left(d_{\mathrm{Co}}\right) / \mathrm{Pt}$ (black a) and $\mathrm{SiO}_{x} / \mathrm{Co}\left(d_{\mathrm{Co}}\right) / \mathrm{Cu}($ red $)$ bilayers [(b), (d), and (f)]. Shown are the average values of 10 fits with different $g$ factor and the maximum and minimum value from these fits.

The anomalous behavior of the bilayers stands out the more when compared to the normal behavior of the trilayers. Both $\mathrm{Cu} / \mathrm{Co}\left(d_{\mathrm{Co}}\right) / \mathrm{Cu}$ and $\mathrm{Pt} / \mathrm{Co}\left(d_{\mathrm{Co}}\right) / \mathrm{Pt}$ show the difference expected for the good spin sink $\mathrm{Pt}$ and the bad spin sink $\mathrm{Cu}$. The angular dependence of $\Delta H_{p p}$ and $H_{r}$ (Fig. 2) and the extracted values for the damping parameter $\alpha$ [Figs. 4(e) and 4(f)] show $\alpha$ to be independent of $d_{\mathrm{Co}}$ in the case of $\mathrm{Cu}$, and increasing with decreasing $d_{\mathrm{Co}}$ in the case of $\mathrm{Pt}$, with more than an order of magnitude difference at the lowest thicknesses. Other parameters of the trilayer also behave in an understandable way. As shown in Fig. 4(c), $4 \pi M_{\text {eff }}$ of the $\mathrm{Pt} / \mathrm{Co}\left(d_{\mathrm{Co}}\right) / \mathrm{Pt}$ trilayer decreases with decreasing $d_{\mathrm{Co}}$, due to the increasing PMA (see Appendix A). For the $\mathrm{Cu}$ case, the decrease is smaller, as expected of the lower PMA of the $\mathrm{Co} / \mathrm{Cu}$ interface [24].

In contrast, the behavior of the $\mathrm{SiO}_{x} / \mathrm{Co} / \mathrm{Cu}$ and $\mathrm{SiO}_{x} / \mathrm{Co} / \mathrm{Pt}$ bilayers does not show the expected behavior. The angular dependence of $\Delta H_{p p}$ and $H_{r}$ (Fig. 2) and the extracted values for the damping parameter $\alpha$ [Fig. 4(f)] show $\alpha$ to be increasing with decreasing $d_{\mathrm{Co}}$ in the case of both $\mathrm{Cu}$ and Pt. Other parameters of the bilayer also do not behave fully understandably. As shown in Fig. 2(a), $4 \pi M_{\text {eff }}$ of the $\mathrm{SiO}_{x} / \mathrm{Co}\left(d_{\mathrm{Co}}\right) / \mathrm{Pt}$ and $\mathrm{SiO}_{x} / \mathrm{Co}\left(d_{\mathrm{Co}}\right) / \mathrm{Cu}$ bilayers both decrease with decreasing $d_{\mathrm{Co}}$. This translates to an increase in PMA for very thin Co thicknesses in the $\mathrm{SiO}_{x} / \mathrm{Co} / \mathrm{Cu}$ bilayer, which is unexpected: the influence of the interfacial anisotropy of the $\mathrm{SiO}_{x} / \mathrm{Co}$ interface is negligible since $K_{s}$ for a $\mathrm{SiO}_{2} / \mathrm{Co}$ interface is of the same order as for a $\mathrm{Co} / \mathrm{Cu}$ interface [25]. It suggests that the increase in $M_{\text {eff }}$ is not due to the PMA $[24,25]$.
We now want to argue that the line broadening observed in the bilayers, and the inferred increase of $\alpha$ with decreasing Co thickness, is an interface effect arising from an extra torque on the electron spins due to the broken symmetry. Such interface effects were first observed by Miron et al. [6] in a Hall-effect measurement on a $\mathrm{Pt} / \mathrm{Co} / \mathrm{AlO}_{x}$ sandwich. There, it was discussed that the interface gives rise to an electric field $\boldsymbol{E}$, which leads to an effective (Rashba) field $\boldsymbol{H}_{R}=\alpha_{R}\left(\hat{\boldsymbol{e}}_{z} \times \boldsymbol{k}\right)$, where $\alpha_{R}$ is a material parameter measuring the strength of the spin-orbit coupling, $\hat{\boldsymbol{e}}_{z}$ is a unit vector parallel to the direction of the $\boldsymbol{E}$ field and perpendicular to the interface, and $\boldsymbol{k}$ is the net electron momentum. In our experiment, $\boldsymbol{k}$ and therefore the average value of $\boldsymbol{H}_{R}$ is zero, but the electrons still move and locally feel the electric field and the effective magnetic field. This results in an increase of the local variations of the effective magnetic field, and the Gilbert damping is proportional to such local variations [14]. The damping will therefore increase. The size of the effective dampinglike field present in the $\mathrm{SiO}_{x} / \mathrm{Co}(2.6) / \mathrm{Pt}$ bilayer corresponds well with the size of the torques found very recently by Hall effect measurements in $\mathrm{AlO}_{\mathrm{x}}(2) / \mathrm{Co}(0.6) / \mathrm{Pt}(3)$ sandwich structures [13].

Ando et al. [26] showed that when a current is sent through the Pt layer, a spin current is generated via the spin Hall effect. This spin current $J_{s}$ can manipulate the magnetization precession. We measured the effect of the injected current on the FMR spectra of a $\mathrm{SiO}_{x} / \mathrm{Co}(5) / \mathrm{Pt}$ bilayer. Even with a current of $40 \mathrm{~mA}$, which would for a $2.4 \times 2.4 \mathrm{~mm}^{2}$ sample result in a current density of approximately $1 \times 10^{9} \mathrm{~A} / \mathrm{m}^{2}$, only a change in $\Delta H_{p p}$ of approximately $5 \%$ was obtained. Note that the Co layer is 5-nm thick. When going to thinner Co films, 
already other mechanisms that influence the magnetization dynamics are more important. Still, the influence of the electric manipulation of the magnetization precession using the SHE does not seem to be the dominant mechanism that results in a large increase of $\Delta H_{p p}$ and $H_{r}$. Furthermore, the same large increase of $\Delta H_{p p}$ and $H_{r}$ is observed in $\mathrm{SiO}_{x} / \mathrm{Co} / \mathrm{Cu}$ bilayers. The spin Hall angle of $\mathrm{Cu}$ is much smaller than the spin Hall angle of $\mathrm{Pt}$, therefore a spin current generated in a $\mathrm{Cu}$ layer due to the SHE will be much smaller than a spin current generated in a Pt layer due to the SHE. The change in $H_{r}$ and $\Delta H_{p p}$ are then expected to be very small in $\mathrm{SiO}_{x} / \mathrm{Co} / \mathrm{Cu}$ bilayer.

In conclusion, we have observed an anomalous increase of the FMR linewidth for thin magnetic Co layers sandwiched asymmetrically between a $\mathrm{Cu}$ or $\mathrm{Pt}$ layer on one side and a $\mathrm{SiO}_{x}$ substrate on the other. We argue this to be due to the Rashba effect at the $\mathrm{SiO}_{x} / \mathrm{Co}$ interface, which gives rise to effective magnetic fields interacting with the electron spins in the Co layer.

\section{ACKNOWLEDGMENTS}

We thank H. Tinkey for help with the experiments, and G. Bauer and S. Bergeret for discussions. Financial support from the European Commission (FP7-ICT-FET No. 225955 STELE) is gratefully acknowledged.

\section{APPENDIX A}

The analysis of the angular dependence of the FMR linewidths $\Delta H_{p p}$ and resonance fields $H_{r}$ yields various parameters which are not essential to the issue of anomalous broadening discussed in the main text, but which nonetheless yield useful information. This concerns the spreads in $\theta_{H}$ and $M_{\text {eff }}$, and the perpendicular anisotropy $K_{\perp}$. They are given below.

\section{1. $\Delta \theta_{H}$ and $\Delta 4 \pi M_{\text {eff }}$}

Figure 5 shows the thickness dependence of the spread in the direction of the easy axis $\Delta\left(\theta_{H}\right)$ (a) and demagnetization field $\Delta\left(4 \pi M_{\text {eff }}\right)$ (b) for the four multilayers, as obtained from analysis of the angular dependence of the FMR. For decreasing Co thicknesses, both $\Delta\left(\theta_{H}\right)$ and $\Delta\left(4 \pi M_{\text {eff }}\right)$ increase for all sets of multilayers.

For both types of trilayers, $\Delta\left(\theta_{H}\right)$ and $\Delta\left(4 \pi M_{\text {eff }}\right)$ becomes larger for very thin Co films. This is expected for very thin Co layers, as the roughness of the $\mathrm{Cu}$ and $\mathrm{Pt}$ buffer layer introduces $\Delta\left(\theta_{H}\right)$ and $\Delta\left(4 \pi M_{\text {eff }}\right)$ of the Co film and the exchange coupling is not strong enough to average out these variations [16]. Although for thicker Co films the roughness of the $\mathrm{Cu}$ and $\mathrm{Pt}$ buffer layer does not change, all magnetic moments in the Co film become parallel to the film plane. For both sets of trilayers, the spread shows the same kind of values as the data set of Mizukami et al. [16], that were used to provide the basis for spin pumping theory [14].

\section{Perpendicular anisotropy}

In Fig. 6(a), the perpendicular anisotropy field $H_{\perp}=$ $4 \pi M_{s}-4 \pi M_{\text {eff }}$, where $H_{\perp}=2 K_{\perp} / M_{s}$, is plotted as a function of the inverse thickness of the Co layer. $H_{\perp}$ is

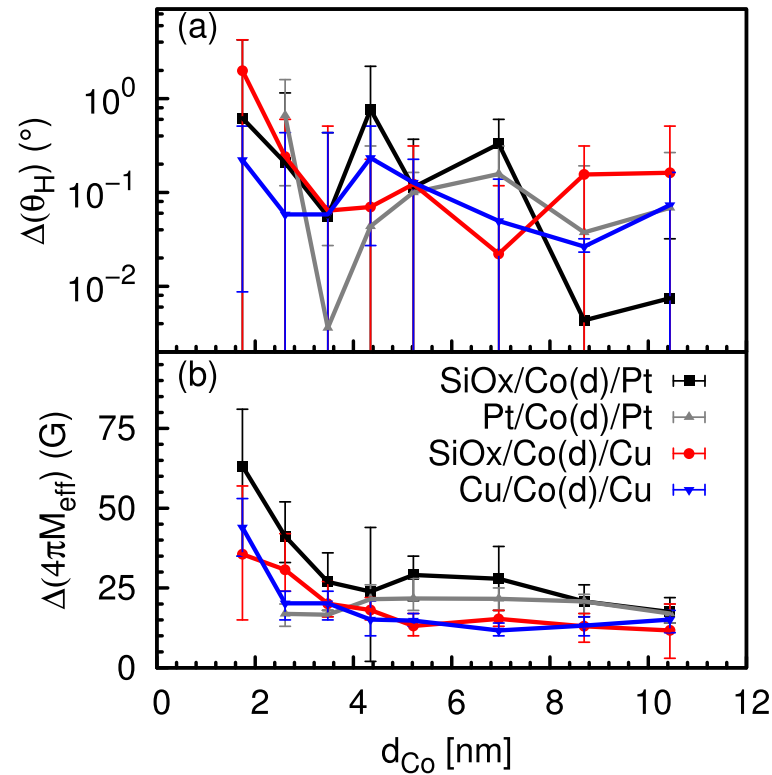

FIG. 5. (Color online) The spatial variations in the direction of the easy axis, $\Delta\left(\theta_{H}\right)$ (a) and the effective demagnetization field, $\Delta\left(4 \pi \mathrm{M}_{\mathrm{eff}}\right)(\mathrm{b})$, as a function of the thickness $d$ of the Co layer in the $\mathrm{SiO}_{x} / \mathrm{Co}(d) / \mathrm{Pt}$ (black $\left.\mathbf{\square}\right), \mathrm{SiO}_{x} / \mathrm{Co}(d) / \mathrm{Cu}$ (red $\bigcirc$ ), $\mathrm{Cu} / \mathrm{Co}(d) / \mathrm{Cu}$ (blue $\boldsymbol{\nabla}$ ) and $\mathrm{Pt} / \operatorname{Co}(d) / \mathrm{Pt}$ (gray $\triangle$ ) multilayers. Shown are the average values of 10 simulations with different $g$ factor and the maximum and minimum value from this simulations.

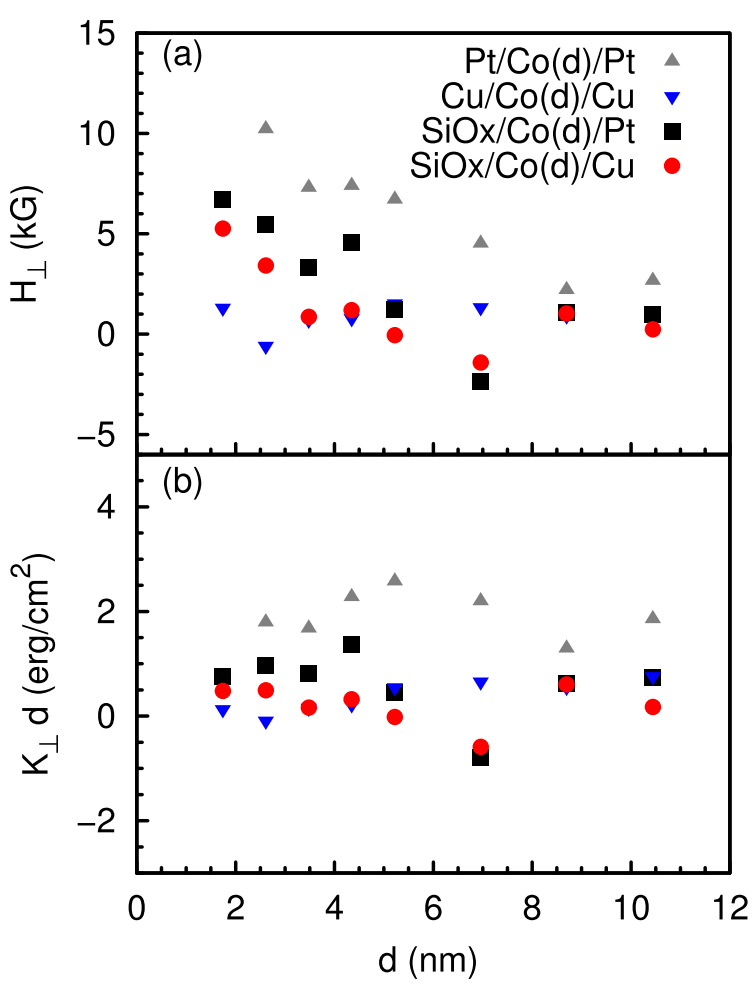

FIG. 6. (Color online) (a) The perpendicular anisotropy field $H_{\perp}$ as a function of the Co thickness $d$. (b) $K_{\perp} d$, with $K_{\perp}$ the anisotropy constant as a function of $d$. In both cases, the multilayers are $\mathrm{Pt} / \mathrm{Co}(d) / \mathrm{Pt}$ (gray $\triangle$ ); $\mathrm{Cu} / \mathrm{Co}(d) / \mathrm{Cu}$ (blue $\nabla$ ); $\mathrm{SiO}_{x} / \mathrm{Co}(d) / \mathrm{Pt}$ (black $\square$ ); and $\mathrm{SiO}_{x} / \mathrm{Co}(d) / \mathrm{Cu}$ (red $)$ ); and multilayers. 
largest in the $\mathrm{Pt} / \mathrm{Co} / \mathrm{Pt}$ trilayer, followed by the $\mathrm{SiO}_{x} / \mathrm{Co} / \mathrm{Pt}$ and $\mathrm{SiO}_{x} / \mathrm{Co} / \mathrm{Cu}$ bilayers, respectively, while $H_{\perp}$ of the $\mathrm{Cu} / \mathrm{Co} / \mathrm{Cu}$ trilayer is quite small. The constant of perpendicular magnetic anisotropy (PMA), $K_{\perp}$, consists of a contribution of the anisotropy of the interface atoms $K_{s}$ and the inner atoms of the magnetic layer $K_{v}$ with thickness $d$ according to

$$
K_{\perp}=K_{v}+2 \frac{K_{s}}{d} .
$$

In Fig. 6(b), $K_{\perp} d$ is plotted as a function of the thickness of the Co layer. Since the value of $K_{v}$ for Co is negative, a linear dependence with a negative slope is expected with, for epitaxial films, a value of the order of $10^{7} \mathrm{erg} / \mathrm{cm}^{3}$ (or $1 \mathrm{MJ} / \mathrm{m}^{2}$ ) [24]. This is not really observed, although at thicknesses above $4 \mathrm{~nm}$ a negative trend starts to set in. The weak development of bulk PMA is probably due to the polycrystalline nature of our sputtered films. On the other hand, it is also well known that the interface PMA at the $\mathrm{Co} / \mathrm{Pt}$ interface is positive and strong, and also that in $\mathrm{Co} / \mathrm{Pt} / \mathrm{Co}$ trilayers the largest contribution for $K_{s}$ comes from the bottom interface [27,28]. The value of $K_{s}$ for a trilayer is roughly $2 \mathrm{erg} / \mathrm{cm}^{2}$, and this coincides very well with the values shown in Fig. 6. The value for the $\mathrm{SiO}_{x} / \mathrm{Co} / \mathrm{Pt}$ bilayer is lower, as expected, while the values for the $\mathrm{Cu}$-based samples are close to zero since the interface PMA for $\mathrm{Cu}$ is weak.

\section{APPENDIX B}

Here, we give $\Delta H_{p p}$ and resonance fields $H_{r}$ for bilayers on other substrates than Si. In Fig. 7, $\Delta H_{p p}$ and $H_{r}$ of a $\mathrm{Co}(3.6) / \mathrm{Cu}$ bilayer grown at room temperature on substrates of single crystal $\mathrm{MgO}$ (cubic, $a=0.421 \mathrm{~nm}$ ), $\mathrm{TiO}_{2}$ (tetragonal, $a=b=0.460 \mathrm{~nm}, c=0.296 \mathrm{~nm}$ ), and $\mathrm{Al}_{2} \mathrm{O}_{3}$ (hexagonal, $a=0.475 \mathrm{~nm}, c=1.299 \mathrm{~nm}$ ) are shown for an in-plane magnetic field $\left(\theta_{H}=90^{\circ}\right)$. Clearly visible in Fig. $7(\mathrm{a})$ is that there is a slight variation in $H_{r}$ for the $\mathrm{SiO}_{x}, \mathrm{MgO}$, and $\mathrm{Al}_{2} \mathrm{O}_{3}$, but $H_{r}$ of the $\mathrm{Co} / \mathrm{Cu}$ bilayer grown on $\mathrm{TiO}_{2}$ and $\mathrm{SiO}_{x} / \mathrm{Cu}$ is much smaller. $\Delta H_{p p}$, as shown in Fig. 7(b), shows a different trend. $\Delta H_{p p}$ of the $\mathrm{Co} / \mathrm{Cu}$ bilayer grown on $\mathrm{SiO}_{x} / \mathrm{Cu}$ has the smallest linewidth, those for the bilayers grown on $\mathrm{TiO}_{2}$ and $\mathrm{Al}_{2} \mathrm{O}_{3}$ are slightly larger, and $\Delta H_{p p}$ of the $\mathrm{Co} / \mathrm{Cu}$ bilayer grown on $\mathrm{MgO}$ is even significantly larger.

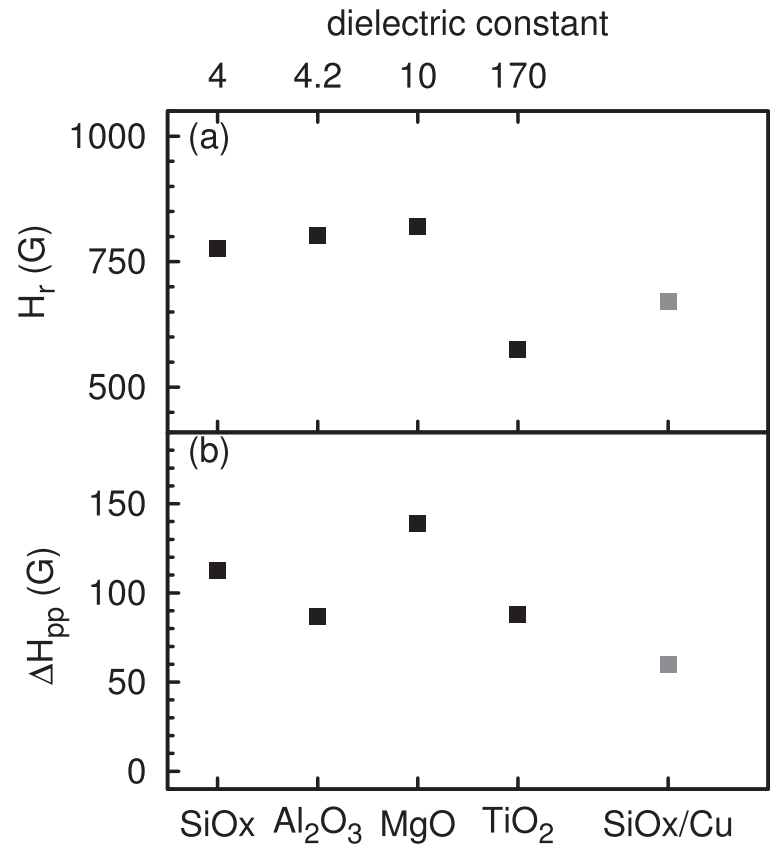

FIG. 7. The resonance field $H_{r}$ (a) and the peak-to-peak linewidth $H_{p p}$ (b) of a $\mathrm{Co}(3.6) / \mathrm{Cu}$ bilayer grown on a single crystal $\mathrm{MgO}, \mathrm{TiO}_{2}$, $\mathrm{Al}_{2} \mathrm{O}_{3}$ for an in-plane magnetic field $\left(\theta_{H}=90^{\circ}\right)$. As a reference, also $\mathrm{Si}$ with a native oxide layer and a $\mathrm{Cu}$ buffer layer (gray symbol) is shown.

All bilayers were grown in the same deposition run, so the sample-to-sample growth variation is very small, but no effort was made to optimize the growth to obtain epitaxial layers. Still, the bilayers probably grow slightly different on each substrate, because of the different lattice constants. Also the interface between the Co and the substrate is different, resulting in a different value for $K_{s}$ and for $M_{\text {eff }}$ and $H_{r}$. Monso et al. [29], Rodmacq et al. [30], and Yang et al. [31] showed that despite the weak spin-orbit interaction at the interface, a PMA is observed for the substrate/Co interface that is comparable to or even larger than the one for a $\mathrm{Co} / \mathrm{Pt}$ or a $\mathrm{Co} / \mathrm{Pd}$ interface. We come to a similar conclusion: all substrate/Co interfaces lead to a significant increase in the damping as signified by the larger FMR linewidth.
[1] A. Chernyshov, M. Overby, X. Liu, J. K. Furdyna, Y. LyandaGeller, and L. P. Rokhinson, Nat. Phys. 5, 656 (2009).

[2] M. Endo, F. Matsukura, and H. Ohno, Appl. Phys. Lett. 97, 222501 (2010).

[3] H. Kurebayashi, Jairo Sinova, D. Fang, A. C. Irvine, T. D. Skinner, J. Wunderlich, V. Novák, R. P. Campion, B. L. Gallagher, E. K. Vehstedt, L. P. Zârbo, K. Výborný, A. J. Ferguson, and T. Jungwirth, Nat. Nanotech. 9, 211 (2014).

[4] X. Z. Yu, N. Kanazawa, Y. Onose, K. Kimoto, W. Z. Zhang, S. Ishiwata, Y. Matsui, and Y. Tokura, Nat. Mater. 10, 106 (2011).

[5] F. Jonietz, S. Mühlbauer, C. Pfleiderer, A. Neubauer, W. Münzer, A. Bauer, T. Adams, R. Georgii, P. Böni, R. A. Duine, K. Everschor, M. Garst, and A. Rosch, Science 330, 1648 (2010).
[6] I. M. Miron, G. Gaudin, S. Auffret, B. Rodmacq, A. Schuhl, S. Pizzini, J. Vogel, and P. Gambardella, Nat. Mater. 9, 230 (2010).

[7] I. M. Miron, K. Garello, G. Gaudin, P.-J. Zermatten, M. V. Costache, S. Auffret, S. Bandiera, B. Rodmacq, A. Schuhl, and P. Gambardella, Nature (London) 476, 189 (2011).

[8] U. H. Pi, K. W. Kim, J. Y. Bae, S. C. Lee, Y. J. Cho, K. S. Kim, and S. Seo, Appl. Phys. Lett. 97, 162507 (2010).

[9] I. M. Miron, T. Moore, H. Szambolics, L. D. Buda-Prejbeanu, S. Auffret, B. Rodmacq, S. Pizzini, J. Vogel, M. Bonfim, A. Schuhl, and G. Gaudin, Nat. Mater. 10, 419 (2011).

[10] C. O. Avci, K. Garello, I. M. Miron, G. Gaudin, S. Auffret, O. Boulle, and P. Gambardella, Appl. Phys. Lett. 100, 212404 (2012). 
[11] K.-W. Kim, S.-M. Seo, J. Ryu, K.-J. Lee, and H.-W. Lee, Phys. Rev. B 85, 180404 (2012).

[12] L. Liu, C.-F. Pai, Y. Li, H. W. Tseng, D. C. Ralph, and R. A. Buhrman, Science 336, 555 (2012).

[13] K. Garello, I. M. Miron, C. O. Avci, F. Freimuth, Y. Mokrousov, S. Blugel, S. Auffret, O. Boulle, G. Gaudin, and P. Gambardella, Nat. Nanotechnol. 8, 587 (2013).

[14] Y. Tserkovnyak, A. Brataas, and G. E. W. Bauer, Phys. Rev. Lett. 88, 117601 (2002).

[15] M. Farle, Rep. Prog. Phys. 61, 755 (1998).

[16] S. Mizukami, Y. Ando, and T. Miyazaki, Jpn. J. Appl. Phys. 40, $580(2001)$

[17] J. Dubowik, K. Załęski, H. Głowiński, and I. Gościańska, Phys. Rev. B 84, 184438 (2011).

[18] H. Suhl, Phys. Rev. 97, 555 (1955).

[19] C. Chappert, K. L. Dang, P. Beauvillain, H. Hurdequint, and D. Renard, Phys. Rev. B 34, 3192 (1986).

[20] W. Platow, A. N. Anisimov, G. L. Dunifer, M. Farle, and K. Baberschke, Phys. Rev. B 58, 5611 (1998).

[21] C. H. Marrows and B. J. Hickey, Phys. Rev. B 63, 220405 (2001).
[22] A. N. Anisimov, M. Farle, P. Poulopoulos, W. Platow, K. Baberschke, P. Isberg, R. Wäppling, A. M. N. Niklasson, and O. Eriksson, Phys. Rev. Lett. 82, 2390 (1999).

[23] Z. Zhang, P. E. Wigen, and S. S. P. Parkin, J. Appl. Phys. 69 5649 (1991).

[24] M. T. Johnson, P. J. H. Bloemen, F. J. A. den Broeder, and J. J. de Vries, Rep. Prog. Phys. 59, 1409 (1996).

[25] J.-G. Zhu, V. Sokalski, Y. Wang, and D. Laughlin, IEEE Trans. Magn. 47, 74 (2011).

[26] K. Ando, S. Takahashi, K. Harii, K. Sasage, J. Ieda, S. Maekawa, and E. Saitoh, Phys. Rev. Lett. 101, 036601 (2008).

[27] S. Bandiera, R. C. Sousa, B. Rodmacq, and B. Dieny, IEEE Mag. Lett. 2, 3000504 (2011).

[28] S. Bandiera, R. C. Sousa, B. Rodmacq, and B. Dieny, Appl. Phys. Lett. 100, 142410 (2012).

[29] S. Monso, B. Rodmacq, S. Auffret, G. Casali, F. Fettar, B. Gilles, B. Dieny, and P. Boyer, Appl. Phys. Lett. 80, 4157 (2002).

[30] B. Rodmacq, S. Auffret, B. Dieny, S. Monso, and P. Boyer, J. Appl. Phys. 93, 7513 (2003).

[31] H. X. Yang, M. Chshiev, B. Dieny, J. H. Lee, A. Manchon, and K. H. Shin, Phys. Rev. B 84, 054401 (2011). 